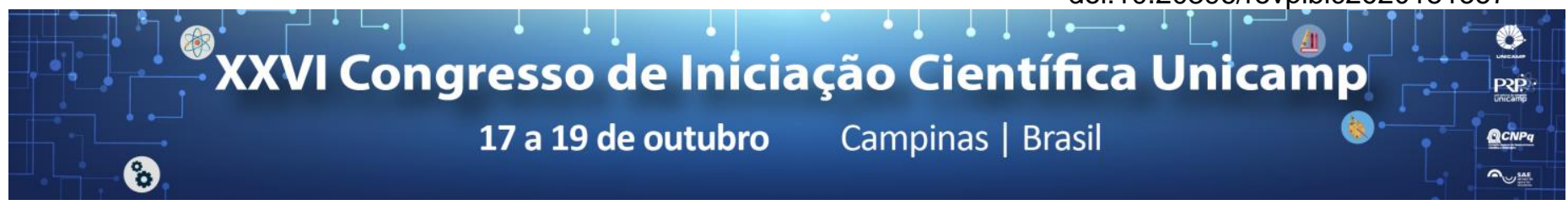

\title{
Development of biomimetic structures through topology optimization
}

\section{Vinicius Mizobuti *, Luiz C. M. Vieira Junior.}

\begin{abstract}
This research project proposed the use of topology optimization as a method for developing biomimetic structures while exploring and evaluating its applications in architectural design. The advancements in computer-aided design tools in recent decades made possible the implementation of computational methods in the design process, enabling the use of geometries as complex as those found in nature, which demands the proper optimization of their structural systems in order to avoid unnecessary costs. Experiments with topology optimization were conducted in multiple 2D and 3D domains, under support and loading cases inspired by nature, and the optimal solutions obtained were compared with images of existing species. The results indicates the validity of the use of topology optimization as a method for conceiving biomimetic structures and as such, its implementation in bioinspired architecture. Future developments of the research includes the implementation of the method as a tool for predicting biological evolutionary processes.
\end{abstract}

\section{Key words:}

biomimetics, topology optimization, structural engineering

\section{Introduction}

Biomimetics is a field of interdisciplinary research where principles of engineering, chemistry and biology are applied in the development of technologies that imitates or are inspired by biological processes. Recent works in the field have demonstrated the use of finite element method as a tool for modeling and evaluating biomimetic structures ${ }^{1}$, but its modeling as a result of optimization processes remains unexplored. Our hypothesis is that structures found in nature could have evolved through a process that is similar to that of topology optimization, where the optimal distribution of an isotropic material in space establishes the topology of a structure, while responding to stresses caused by its environment ${ }^{2}$. Understanding the parameters that have influenced the structural design in existing species will allow the translation of those solutions at different scales in bioinspired architecture, while inevitably creating opportunities to explore the use of topology optimization in predicting future biological evolutionary processes.

\section{Results and Discussion}

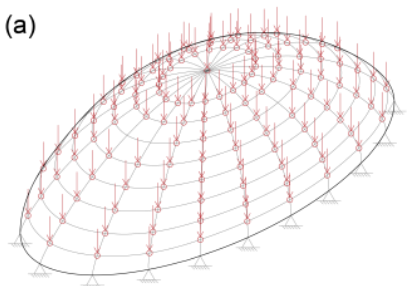

(b)

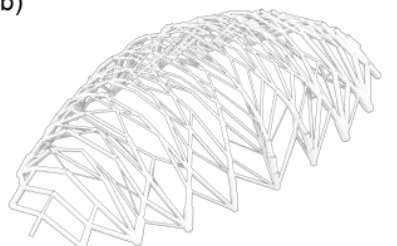

(c)

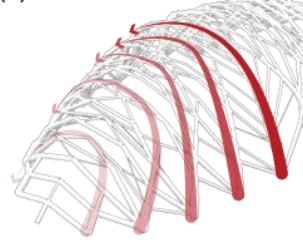

(d)

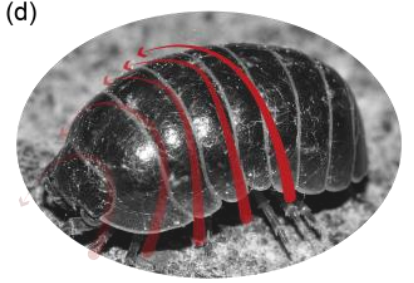

Image 1. (a) Elipsoid domain (b) Optimal structure (c) Main components (d) Comparison with Armadillidium vulgare ${ }^{5}$

The experiments were implemented through MATLAB codes $^{3,4}$, and were based on plant leaves for the $2 \mathrm{D}$ case and animal shells for the $3 \mathrm{D}$ case due to their relative symmetry. A sample of the optimal solutions obtained and their respective comparison with an existing species is presented in Image 1. While the 2D experiments relied on the domains provided by the original code, the $3 \mathrm{D}$ experiments made use of an external 3D parametric modeling software for both the development of new domains and for an improved visualization and rendering of the results.

\section{Conclusions}

Through the results we were able to conclude that topology optimization is a method capable of conceiving biomimetic structures and thus, its scalability to bioinspired architecture under analagous environmental conditions. Further developments of this research includes the use of this optimization method in the evaluation and prediction of biological evolutionary processes, demanding the modeling of more detailed domains and loading conditions to properly represent the current and future environmental scenarios.

\section{Acknowledgement}

The authors aknowledges the National Council for Scientific and Technological Development (CNPq) for their financial support through the PIBIC scholarship.

\footnotetext{
1 Mengesha, T. E. et al. Parametric structural modeling of insect wings. Bioinspiration \& Biomimetics, v.4, n.3, 2009.

${ }^{2}$ Bendsoe, E. M. P. Simung, O. Topology Optimization: Theory, Methods and Applications. 2002.

3 Zegard, T. Paulino G. H. GRAND: Ground structure based topology optimization for arbitrary 2D domains using MATLAB. Structural and Multidisciplinary Optimization, v.50, n.5, 2014.

4 Zegard, T. Paulino G. H. GRAND3: Ground structure based topology optimization for arbitrary 3D domains using MATLAB. Structural and Multidisciplinary Optimization, v.52, n.5, 2015.

5 Welter-Schultes, F. Family summary for Armadillidiidae. Disponível em <www.animalbase.uni-goettingen.de>. Acesso em: 15 jul. 2018.
} 